\title{
Política tecnológica y cooperación en I+D: una visión a través de un modelo formal*
}

\author{
Antonio García-Lorenzo y José López-Rodríguez ${ }^{* *}$
}

\begin{abstract}
Resumen
La mayor parte de la literatura empírica ha mostrado que el apoyo público a las actividades de I $+\mathrm{D}$ empresariales estimula la cooperación. Sin embargo, no hay un modelo formal que estudie la relación entre la política tecnológica y la cooperación en $\mathrm{I}+\mathrm{D}$. El objetivo de este trabajo es desarrollar un modelo principal-agente que permita determinar la estructura de incentivos que el principal —el gobierno como responsable de la elaboración de los programas de política tecnológica - otorga a los agentes - las empresas, las universidades y otros organismos públicos de investigación que forman parte del sistema- para que cooperen en $\mathrm{I}+\mathrm{D}$. Los resultados muestran que a partir de un determinado umbral de incentivos se fomenta la cooperación. En lo que se refiere a la política tecnológica, la principal implicación práctica de este resultado es que las ayudas públicas a la $\mathrm{I}+\mathrm{D}$ pueden provocar un cambio en el enfoque u organización de la estrategia de I+D empresarial —cooperación versus no cooperación-, que dependerá de la cuantía de las ayudas recibidas. La principal implicación teórica de este trabajo es que la nueva metodología - modelo principal-agente- puede proporcionarnos un marco útil para analizar los efectos de la política tecnológica sobre la cooperación.
\end{abstract}

Palabras clave: ayudas públicas a la $\mathrm{I}+\mathrm{D}$, cooperación en $\mathrm{I}+\mathrm{D}$, política tecnológica, modelo principal-agente.

Clasificación JEL: D86, H32, O31, O38.

\section{INTRODUCCIÓN}

La cooperación en investigación y desarrollo (I+D) emerge como una importante estrategia empresarial en las últimas tres décadas en los países de la Organización para la Cooperación y el Desarrollo Económicos (OCDE) (Hagedoorn,

Recibido el 28 de febrero del 2014; aceptado el 21 de mayo del 2015.

* Una versión preliminar de este trabajo está publicada en inglés en la Fundación de las Cajas de Ahorros (Funcas), Working Paper no. 727, septiembre de 2013.

** Departamento de Análisis Económico y ADE, Facultad de Economía y Empresa de la Universidade da Coruña (España),<aglec@udc.es>y <jolopez@udc.es> respectivamente. Los autores agradecen los valiosos comentarios de los dictaminadores anónimos de la revista. 
2002; OECD, 2002; 2010). Al mismo tiempo, los gobiernos han promovido la cooperación tecnológica con el objetivo de: a) corregir los fallos de mercado en las inversiones en $\mathrm{I}+\mathrm{D}$, en particular, cuando exista una elevada dificultad para la apropiación de los resultados; ${ }^{1}$ b) acelerar la innovación tecnológica para incrementar la competitividad internacional, $\mathrm{y} c$ ) incrementar el intercambio de información tecnológica entre empresas, universidades y organismos públicos de investigación (OPI’s) ${ }^{2}$ (Hagedoorn, Link y Vonortas, 2000).

Sin embargo, como consecuencia de la intervención pública surge una cuestión importante relacionada con la evaluación expost de estas políticas tecnológicas, con el objetivo de averiguar si las políticas de apoyo a la cooperación en $\mathrm{I}+\mathrm{D}$ afectan al comportamiento estratégico empresarial, tanto en términos de cooperación como de funcionamiento de dichas actividades. La principal cuestión es si estos resultados podrían haberse alcanzado igualmente sin dicho apoyo y, por tanto, si esta política de intervención debería mantenerse. En este sentido, varios estudios empíricos han analizado el impacto de los programas de ayudas públicas a la $\mathrm{I}+\mathrm{D}$ en la colaboración con el fin de evaluar si la participación en estos programas incrementa el número de acuerdos y, en consecuencia, si el apoyo público sitúa la cooperación por encima del nivel en el que el mercado la habría situado sin tal apoyo (lo que es conocido en la literatura como adicionalidad en el comportamiento). ${ }^{3,4}$ La evidencia empírica muestra que, en general, los

1 La justificación del apoyo público a las actividades de $\mathrm{I}+\mathrm{D}$ reside en el argumento tradicional de que los fallos de mercado reducen los incentivos privados a las inversiones en $\mathrm{I}+\mathrm{D}$, situando dichas inversiones en niveles por debajo de lo socialmente deseable (subóptimo social). Este argumento también puede utilizarse para justificar el apoyo público a la cooperación en estas actividades.

2 Algunos ejemplos de programas específicos que fomentan la cooperación son los siguientes: Engineering Research Associations en Japón, el Advanced Technology Program en Estados Unidos, y los sucesivos Framework Programs on Research and Technological Development en la Unión Europea.

3 En la literatura existente sobre la evaluación de la política tecnológica, la adicionalidad en un sentido amplio significa que los programas públicos de $\mathrm{I}+\mathrm{D}$ contribuyen a crear un bienestar adicional que no se hubiera conseguido sin dicha intervención (Buisseret, Cameron y Georghiou, 1995; Davenport, Grimes y Davies, 1998). Dado que los efectos sobre el bienestar son difíciles de medir, existen otros indicadores de adicionalidad. Estos son, adicionalidad en inputs, outputs y comportamental. La adicionalidad de los imputs se refiere a los cambios en los gastos privados motivados por el apoyo público. La adicionalidad en el output hace referencia a los cambios en las patentes y nuevos productos obtenidos por el apoyo recibido por la empresa. La adicionalidad en el comportamiento se refiere a los cambios en las estrategias de colaboración empresarial debidos a la participación en los programas públicos de I+D.

4 La mayoría de la literatura empírica que evalúa los programas públicos de $\mathrm{I}+\mathrm{D}$ ha centrado su atención en el efecto que éstos tienen sobre el gasto privado, las patentes y otras medidas de resultados, 
fondos públicos de ayuda a la $\mathrm{I}+\mathrm{D}$ estimulan el aumento de la cooperación. No obstante, es importante señalar algunos problemas metodológicos que se ponen de manifiesto en diferentes estudios (e.g., Bayona, García-Marco y Huerta, 2003; Fölster, 1995; Miotti y Sachwald, 2003; Mohnen y Hoareau, 2003) y que están relacionados con la endogeneidad de los subsidios y las características de los programas de I+D.

El problema de endogeneidad se origina porque la participación en los programas públicos de $\mathrm{I}+\mathrm{D}$ y la cooperación pueden darse simultáneamente debido a que existen factores que se omiten en el programa de participación, tales como las características del proyecto, que afectan la decisión de cooperación y, en consecuencia, sobreestiman los resultados. ${ }^{5}$ Respecto a las características de los programas de $\mathrm{I}+\mathrm{D}$, hay algunos programas (e.g., programas de la Unión Europea) en los cuales la cooperación es una exigencia para obtener fondos, de tal forma que la participación en tales programas y la cooperación no son elecciones diferentes. Algunos trabajos empíricos (Belderbos, Carreeb y Lokshinb, 2004; Buson y Fernández-Ribas, 2008) han considerado explícitamente estos problemas mostrando alguna divergencia en sus resultados. Belderbos, Carreeb y Lokshinb (2004) muestran que el efecto derivado de los subsidios a la I+D no es concluyente, dado que los resultados varían con la estrategia empírica usada para controlar la endogeneidad, ${ }^{6}$ mientras que Buson y Fernández-Ribas (2008) constatan que los programas nacionales de $\mathrm{I}+\mathrm{D}$ tienen un efecto positivo sobre la cooperación vertical privada y especialmente en la cooperación públicoprivada. Por lo tanto, la conclusión es que, aunque los resultados muestran una tendencia positiva del efecto de los subsidios a la I+D sobre la cooperación, debe ponerse un especial cuidado en el tratamiento de la endogeneidad de los subsidios de $\mathrm{I}+\mathrm{D}$, explorando procedimientos alternativos que nos permitan asegurar la robustez de los resultados.

pero no en cómo se organizan estas actividades — colaboración versus no colaboración- (Buson y Fernández-Ribas, 2008).

5 En términos matemáticos, el problema de endogeneidad aparece cuando alguna de las variables independientes de la ecuación de regresión está correlacionada con el término de error y, por tanto, los estimadores de mínimos cuadrados ordinarios (MCO) son inconsistentes.

6 Cuando se utilizan retardos en los subsidios muestran un efecto positivo en la probabilidad de cooperación vertical y público-privada, pero no en la cooperación horizontal. Sin embargo, cuando restringimos la muestra para incluir solamente empresas que son nuevas en la cooperación, los subsidios a la I+D no tienen ningún efecto sobre la cooperación vertical o pública y, además, tienen un efecto negativo sobre la cooperación horizontal. 
En este contexto, nuestro trabajo pretende ampliar esta senda de investigación mediante el desarrollo de un modelo formal que incorpore el papel de la política tecnológica sobre la cooperación entre los agentes del sistema. Para ello, considerando la relación que se establece entre el principal —el gobierno como responsable del diseño de la política tecnológica-y los agentes —empresas, universidades y Opı's- como una relación de agencia, se elabora un modelo básico principal-agente mediante el cual se deriva la estructura óptima de incentivos que el gobierno debe proveer a los agentes para que les induzca a la cooperación en el desarrollo de las actividades de I+D. Se consideran dos entornos; por un lado, una situación en la cual los agentes actúan de manera individual y que nos sirve de benchmarking y, por otro lado, una situación en la que los agentes actúan de manera cooperativa. Nuestros resultados muestran que existe un nivel de incentivos que promueven la cooperación debido a que cada agente alcanza un nivel de utilidad superior cuando coopera en comparación al que alcanzaría en la situación de no cooperación. La principal implicación práctica/política de este resultado es que los subsidios a la $\mathrm{I}+\mathrm{D}$ pueden estimular a los agentes a la cooperación y que esto dependerá de su cuantía. Desde el punto de vista teórico, nuestro trabajo presenta una nueva metodología basada en el uso de modelos formales para el análisis del efecto de la política tecnológica en la cooperación que contribuirá a una mejor comprensión de este fenómeno.

El resto del trabajo se estructura como sigue. En la sección dos se introduce el marco analítico básico del modelo principal-agente. En la tres se estudian las soluciones que el principal -i.e., el contrato de incentivos- ofrece a los agentes considerando dos modelos: a) modelo 1 , en el que cada agente realiza actividades de I+D de manera individual - modelo no cooperativo- y b) modelo 2 , en el que cada agente colabora con otros en la realización de actividades de $\mathrm{I}+\mathrm{D}$-modelo cooperativo-. Mediante la comparación de ambas soluciones, podemos determinar la estructura de incentivos que promueven la cooperación. Finalmente, en la sección cuatro se presentan las conclusiones, implicaciones y futuras líneas de investigación.

\section{El MARCo analítico}

Consideramos la relación que se establece entre el gobierno y los agentes a través de la política tecnológica como una relación de agencia, en la que el gobierno, actuando como principal, diseña el contrato de incentivos —el programa de política 
tecnológica - que ofrece a los agentes para estimular el desarrollo de actividades de I+D. Dicha relación se formaliza mediante un modelo principal-agente y en un contexto de información asimétrica sobre el nivel de esfuerzo del agente. Formalmente, se asume que la función de utilidad del gobierno - función de utilidad social— puede expresarse como sigue:

$$
U(R, W)=B(R)-v(W)
$$

donde $B(R)$ representa el beneficio que el gobierno obtiene de los resultados derivados de la aplicación de la política tecnológica (número de innovaciones o patentes que implican un mayor nivel de desarrollo tecnológico) y $v(W)$ representa el costo o la desutilidad asociada a los incentivos que el gobierno ofrece a los agentes para desarrollar sus actividades de I+D. Matemáticamente, las funciones exhiben el comportamiento siguiente: $B^{\prime}>0, B^{\prime \prime} \leq 0, v^{\prime}>0 \mathrm{y}$ $v^{\prime \prime} \geq 0$.

La función de utilidad de los agentes innovadores puede expresarse como:

$$
U(W, E)=u(W)-v(E)
$$

donde $u(W)$ es la utilidad derivada de los incentivos que el gobierno le ofrece y $v(E)$ representa el costeo o la desutilidad asociada al esfuerzo en $\mathrm{I}+\mathrm{D}$ que asume el agente; $u^{\prime}(\cdot)>0, u^{\prime \prime}(\cdot) \leq 0, v^{\prime}>0$ y $v^{\prime \prime} \geq 0$.

Formalmente, asumimos que hay varios posibles niveles de resultados (en nuestro caso resultados de las actividades de $\mathrm{I}+\mathrm{D}$ ) que el principal puede esperar -en la sección siguiente, estos posibles resultados serán especificados a partir de la determinación de las soluciones del principal—y, asimismo, se identifican las posibles acciones que un agente puede adoptar teniendo en cuenta que cada acción conlleva un costo. De esta manera, se asume, en general, que el agente puede escoger entre dos niveles posibles de esfuerzo: esfuerzo alto $\left(E^{H}\right)$ y esfuerzo bajo $\left(E^{L}\right)$; y que proveer un esfuerzo alto es más costoso que adoptar un esfuerzo más bajo. También se asume que el principal no puede observar las acciones del agente - i.e., el esfuerzo realizado-, pero si los resultados, lo que técnicamente se considera como un modelo con información asimétrica sobre el esfuerzo del agente. Para que este supuesto tenga sentido, cada acción puede no corresponderse con un determinado nivel de resultado, dado que en este caso, observando el resultado, se advierten también las acciones del 
agente. Por lo tanto, cada una de las posibles acciones de los agentes $-E^{H}$ y $E^{L}$ - se corresponden con una determinada distribución de probabilidad con independencia del nivel de resultados. En la sección siguiente se detallan estas distribuciones de probabilidad.

En este tipo de modelos se supone que hay un cierto nivel de "utilidad de reserva" $(\underline{U})$ para el agente. Este nivel de utilidad representa la utilidad que el agente debe recibir en su mejor alternativa posible. En el proceso de diseño del esquema de incentivos, el principal se enfrenta a dos tipos de restricciones. La primera se refiere a la restricción de participación: si el principal quiere que el agente elija una determinada opción —generalmente se asume que el principal prefiere que el agente ofrezca un nivel de esfuerzo alto en lugar de bajo- la utilidad esperada del agente ha de ser como mínimo igual a la utilidad de reserva. La segunda restricción es la restricción de compatibilidad de incentivos: la diferencia entre la utilidad esperada de los pagos recibidos y el costo asociado a un nivel de esfuerzo alto ha de ser mayor o igual que la asociada a un nivel de esfuerzo bajo. En otras palabras, esto implica que el agente ha de recibir mayor compensación a medida que su esfuerzo se incrementa.

Teniendo en cuenta estos rasgos básicos, nuestro principal objetivo es analizar la estructura de incentivos que el gobierno debe otorgar a los agentes para fomentar la cooperación. A nivel metodológico, consideramos dos escenarios para las actividades de $\mathrm{I}+\mathrm{D}$, uno en el que el agente desarrolla estas actividades individualmente - modelo $1-$ y que nos sirve como benchmarking frente a la situación en la que las actividades de $\mathrm{I}+\mathrm{D}$ se desarrollan de manera cooperativa entre dos agentes - modelo 2-. La comparación entre los niveles de utilidad esperada por los agentes en ambas situaciones nos permite determinar la estructura de incentivos que promueve la cooperación.

\section{SOLUCIONES DEL MODELO}

\section{Las actividades de I+D son desarrolladas individualmente (modelo 1)}

De acuerdo con los comentarios realizados en la sección previa, suponemos que se pueden identificar dos posibles resultados: resultado alto $\left(R^{H}\right)$ y resultado bajo $\left(R^{L}\right)$. La frecuencia con la que se presentan los resultados alto y bajo va a depender del esfuerzo en actividades de I+D que asuma el agente $(E)$ y de otras 
variables aleatorias del entorno. Teniendo en cuenta lo anterior, se establecen las siguientes distribuciones de probabilidad:

$\operatorname{Prob}\left[R=R^{H} \mid E^{H}\right]=p^{H}$

$\operatorname{Prob}\left[R=R^{H} \mid E^{L}\right]=p^{L}$

$\operatorname{Prob}\left[R=R^{L} \mid E^{H}\right]=1-p^{H}$

$\operatorname{Prob}\left[R=R^{L} \mid E^{L}\right]=1-p^{L}$

en las que $p^{H}>p^{L}$.

El contrato que el gobierno ofrece a cada uno de los agentes se puede definir de la siguiente manera:

$W^{H}$, si $R=R^{H}$

$W^{L}$, si $R=R^{L}$

donde $W^{H}$ y $W^{L}$ representan los niveles de incentivos altos y bajos, respectivamente, que el agente puede obtener de acuerdo con el resultado obtenido. Los incentivos que ofrecerá el gobierno serán crecientes con el resultado $(R)$ y éste tiende a mejorar a medida que el agente asume un mayor esfuerzo. Basado en esta premisa, la utilidad esperada del agente, como función de su nivel de esfuerzo, puede reflejarse como sigue:

$$
E U^{A}\left(E=E^{H}\right)=p^{H} W^{H}+\left(1-p^{H}\right) W^{L}-v\left(E^{H}\right)
$$

para un nivel de esfuerzo alto.

$$
E U^{A}\left(E=E^{L}\right)=p^{L} W^{H}+\left(1-p^{L}\right) W^{L}-v\left(E^{L}\right)
$$

para un nivel de esfuerzo bajo.

Para calcular el contrato óptimo, el gobierno ha de dar solución al siguiente programa - asumiendo que prefiere que el agente realice un nivel de esfuerzo alto en el desarrollo de sus actividades-:

$$
\begin{gathered}
\operatorname{Min}_{\left[W^{H}, W^{L}\right]} p^{H} v\left(W^{H}\right)+\left(1-p^{H}\right) v\left(W^{L}\right), \text { tal que } \\
p^{H} W^{H}+\left(1-p^{H}\right) W^{L}-v\left(E^{H}\right) \geq \underline{U} \\
p^{H} W^{H}+\left(1-p^{H}\right) W^{L}-v\left(E^{H}\right) \geq p^{L} W^{L}+\left(1-p^{L}\right) W^{L}-v\left(E^{L}\right)
\end{gathered}
$$


donde las ecuaciones [6] y [7] reflejan la restricción de participación y de incentivos respectivamente. ${ }^{7}$ La restricción de participación recoge el hecho de que el agente podría rechazar el contrato si la utilidad esperada que obtiene de ese programa de ayudas no es por lo menos igual a la de reserva. La restricción de incentivos refleja que el agente estaría dispuesto a seleccionar la opción que el gobierno le propone $\left(E=E^{H}\right)$ si dicha opción maximiza su función objetivo, esto es, $E U^{A}=\left(E=E^{H}\right) \geq E U^{A}=\left(E=E^{L}\right)$, lo que supone recibir mayores incentivos. Adicionalmente, teniendo en cuenta que esta restricción será más fuerte en el caso particular en el que el esfuerzo es nulo $\left(E=E^{L}=0\right)$, la ecuación [7] puede rescribirse del modo siguiente:

$$
\left(p^{H}-p^{L}\right)\left(W^{H}-W^{L}\right) \geq v\left(E^{H}\right)
$$

Llamando $\mu$ al multiplicador de la condición de participación y $\lambda$ al de la restricción de incentivos, las condiciones de primer orden del Lagrangiano se escriben como:

$$
\begin{gathered}
p^{H} v^{\prime}\left(W^{H}\right)-p^{H} \mu-\left(p^{H}-p^{L}\right) \lambda=0 \Leftrightarrow v^{\prime}\left(W^{H}\right)=\mu+\frac{p^{H}-p^{L}}{p^{H}} \lambda \\
\left(1-p^{H}\right) v^{\prime}\left(W^{L}\right)-\left(1-p^{H}\right) \mu-\left(p^{H}-p^{L}\right) \lambda=0 \Leftrightarrow v^{\prime}\left(W^{L}\right)=\mu-\frac{p^{H}-p^{L}}{1-p^{H}} \lambda
\end{gathered}
$$

donde $W^{H}$ y $W^{L}$ denotan los incentivos que el agente espera obtener bajo el contrato óptimo si $R=R^{H}$ y $R=R^{L}$ respectivamente.

Dado que $1>p^{H}>p^{L}>0$, y teniendo en cuenta que la función $v(E)$ tomará valores positivos pues representa la desutilidad asociada al esfuerzo, la ecuación [8] implica que $W^{H}>W^{L}$. Por otro lado, si tenemos en cuenta que $v(W)$ es creciente, se cumplirá que $v^{\prime}\left(W^{H}\right)>v^{\prime}\left(W^{L}\right)$. Finalmente, las ecuaciones [9] y [10] implican, en particular, que $\lambda>0$ y $\mu>0$, con lo que las dos restricciones están saturadas. En consecuencia, las restricciones de participación y de incentivos caracterizarán el contrato óptimo, ambas en igualdad.

7 Un método para resolver los programas de optimización con restricciones es el método del multiplicador de Lagrange (Nicholson, 2005). 
Resolviendo el sistema compuesto por las ecuaciones [6] y [8], obtenemos que la solución al programa establecido por el gobierno para diseñar el contrato óptimo se puede definir de la forma siguiente:

$$
\begin{aligned}
& \bar{W}^{H}=\underline{U}+v\left(E^{H}\right) \frac{1-p^{L}}{p^{H}-p^{L}} \\
& \bar{W}^{L}=\underline{U}-v\left(E^{H}\right) \frac{p^{L}}{p^{H}-p^{L}}
\end{aligned}
$$

donde $\bar{W}^{H}$ y $\bar{W}^{L}$ representan los incentivos óptimos que el gobierno debe proporcionar a los agentes innovadores en las situaciones de esfuerzo alto y bajo respectivamente. A partir de estos resultados, se verifica que la utilidad esperada por el agente bajo una hipotética situación de esfuerzo alto, considerando los incentivos establecidos por el gobierno y la ecuación [2], es igual a la utilidad de reserva - se cumple, por tanto, la restricción de participación.

$$
E U^{A}\left(E=E^{H}\right)=p^{H} \bar{W}^{H}+\left(1-p^{H}\right) \bar{W}^{L}-v\left(E^{H}\right)=\underline{U}+v\left(E^{H}\right)-v\left(E^{H}\right)=\underline{U}
$$

Asimismo, también se comprueba que en el caso límite, en el que el resultado observado es alto $\left(R=R^{H}\right)$ y el esfuerzo del agente puede ser correctamente estimado $\left(E=E^{H}\right)$, esto es $p^{H}=1$, los incentivos recibidos por el agente cubrirán su utilidad de reserva y le compensarán, además, por la desutilidad asociada al nivel de esfuerzo asumido. En definitiva, el gobierno ofrecerá al agente unos incentivos que vendrán dados por la expresión siguiente: $\bar{W}^{H}=\underline{U}+v\left(E^{H}\right)$.

\section{Las actividades de $\mathrm{I}+\mathrm{D}$ son desarrolladas cooperativamente (modelo 2 )}

Con el objetivo de determinar la estructura de incentivos que el gobierno debe ofrecer a los agentes si deciden cooperar, estudiaremos en este apartado el programa que el gobierno debería resolver teniendo en cuenta la situación en la que los agentes cooperan - modelo en el que las actividades de $\mathrm{I}+\mathrm{D}$ se realizan de manera cooperativa por varios agentes-, de modo que la utilidad 
esperada por los agentes se compare con la utilidad esperada en el modelo 1. Se resuelve en el modelo el caso más simple de cooperación: dos agentes. Hay que tener en cuenta que los resultados de $\mathrm{I}+\mathrm{D}$ dependerán del esfuerzo conjunto que realicen los agentes participantes en el programa y, al igual que en el modelo 1, se asume que cada uno de los agentes elegirá entre un nivel de esfuerzo alto, $E^{H}$, y un nivel de esfuerzo bajo, $E^{L}$. Cuando los agentes actúan de manera cooperativa se identifican tres posibles resultados: alto $\left(R^{H H}\right)$, bajo $\left(R^{L L}\right)$ e intermedio $\left(R^{L H}\right)$, el cual es inferior a $R^{H H}$ y superior a $R^{L L}$.

La inclusión de este último resultado es consecuencia de los posibles problemas que surgen durante la cooperación, asociados al potencial oportunismo en el esfuerzo realizado por el agente. En otras palabras, un agente puede realizar un esfuerzo bajo - comportamiento oportunista- mientras que el otro realiza un esfuerzo alto. El doble superíndice en la formulación de este tipo de resultado se debe a la presencia de dos agentes en la configuración del modelo cooperativo.

Las nuevas probabilidades asociadas a los posibles resultados que el gobierno obtiene de su relación con los agentes en un contexto de cooperación pueden definirse de la manera siguiente:

Prob $\left[R=R^{H H} \mid E_{1}=E_{1}{ }^{H}, E_{2}=E_{2}{ }^{H}\right]=p_{H}{ }^{2}$

$\operatorname{Prob}\left[R=R^{L L} \mid E_{1}=E_{1}{ }^{H}, E_{2}=E_{2}{ }^{H}\right]=\left(1-p_{H}\right)^{2}$

Prob $\left[R=R^{L} \mid E_{1}=E_{1}{ }^{H}, E_{2}=E_{2}{ }^{L} ; E_{1}=E_{1}{ }^{L}, E_{2}=E_{2}{ }^{L}\right]=2 p^{H}\left(1-p_{H}\right)$

El posible contrato que el gobierno ofrece a los agentes cuando cooperan se define de la manera siguiente:

$$
\begin{aligned}
& W=W_{i}^{H H} ; W=W_{1}^{H} ; W=W_{2}^{H}, \text { si } R=R^{H H} \\
& W=W_{i}^{L L} ; W=W_{1}^{L} ; W=W_{2}^{L} \text {, si } R=R^{L L} \\
& W=W_{i}^{L H} ; W=W_{1}^{L H} ; W=W_{2}^{L H} \text {, si } R=R^{L H}
\end{aligned}
$$

donde $W^{H H}$ representa el incentivo alto que cada uno de los dos agentes recibirá en el caso de que el resultado sea alto y es el equivalente al incentivo $W^{H}$ en el modelo 1 ; idem para $W^{L L}$; mientras que $W^{L H}$ representa el incentivo que cada uno de los agentes recibirá cuando el resultado sea $R^{L H}$. A partir de las ecuaciones anteriores, y teniendo en cuenta las soluciones derivadas en el modelo 1, la utilidad esperada de uno de los agentes en función de su esfuerzo y de 
los incentivos que recibe del gobierno puede expresarse de la manera siguiente - considerando que realiza un nivel de esfuerzo alto-:

$$
E U^{A}\left(E=E^{H}\right)=p_{H}{ }^{2} \bar{W}^{H}+2 p_{H}\left(1-p_{H}\right) W^{L H}+\left(1-p_{H}\right)^{2} \bar{W}^{L}-v\left(E^{H}\right)
$$

Con la sustitución de $\bar{W}^{H}$ y $\bar{W}^{L}$ por los valores obtenidos en la condición de primer orden, se obtiene:

$$
\begin{aligned}
E U^{A}\left(E=E^{H}\right)= & p_{H}{ }^{2}\left(\underline{U}+v\left(E^{H}\right) \frac{1-p_{L}}{p_{H}-p_{L}}\right)+2 p_{H}\left(1-p_{H}\right) W^{L H} \\
& +\left(1-p_{H}\right)^{2}\left(\underline{U}-v\left(E^{H}\right) \frac{p_{L}}{p_{H}-p_{L}}\right)-v\left(E^{H}\right)
\end{aligned}
$$

Definiendo $\bar{W}=\frac{\bar{W}^{H}+\bar{W}^{L}}{2}$ y sumando y restando $\bar{W}$ en la ecuación [14], obtenemos la expresión siguiente:

$$
\begin{aligned}
E U^{A}\left(E=E^{H}\right)= & p_{H}^{2}\left(\underline{U}+v\left(E^{H}\right) \frac{1-p_{L}}{p_{H}-p_{L}}\right)+2 p_{H}\left(1-p_{H}\right)\left(W^{L H}+\bar{W}-\bar{W}\right) \\
& +\left(1-p_{H}\right)^{2}\left(\underline{U}-v\left(E^{H}\right) \frac{p_{L}}{p_{H}-p_{L}}\right)-v\left(E^{H}\right) \\
E U^{A}\left(E=E^{H}\right) & =\underline{U}+v\left(E^{H}\right)+2\left(W^{L H}-\bar{W}\right) p_{H}\left(1-p_{H}\right)-v\left(E^{H}\right) \\
& =\underline{U}+2\left(W^{L H}-\bar{W}\right) p_{H}\left(1-p_{H}\right)
\end{aligned}
$$

La ecuación [16] refleja la utilidad esperada de los agentes cuando cooperan y bajo una situación de esfuerzo alto. A partir de esta expresión, y comparándola con la de la utilidad esperada del agente cuando las actividades de I+D se realizan individualmente, podemos concluir que el valor de la utilidad esperada cuando se coopera depende del nivel de incentivos $W^{\mathrm{LH}}$ que los agentes reciben cuando el resultado es $R^{L H}$. De esta forma, podemos formular la siguiente proposición: 
Proposición. Existe un determinado nivel de incentivos, $W^{L H}>\bar{W}$, a partir del cual la utilidad esperada de los agentes innovadores cuando actúan de manera cooperativa es mayor que la utilidad esperada de los agentes cuando actúan de manera individual. Por lo tanto, bajo esta estructura de incentivos, se promueve la cooperación en actividades de I+D.

Prueba. Se comprueba de manera inmediata que cuando $W^{L H}>\bar{W}$, la utilidad esperada por los agentes - para un nivel de esfuerzo alto- cuando actúan cooperativamente se sitúa por encima de la utilidad de reserva y, por tanto, es mayor que cuando actúan independientemente, pues, en este caso, se limita solamente a la de reserva (modelo 1).

La utilidad de los agentes cuando actúan independientemente será:

$$
E U^{A}\left(E=E^{H}\right)=p^{H} \bar{W}^{H}+\left(1-p^{H}\right) \bar{W}^{L}-v\left(E^{H}\right)=\underline{U}+v\left(E^{H}\right)-v\left(E^{H}\right)=\underline{U}
$$

Mientras que la utilidad cuando actúan de manera cooperativa será:

$$
E U^{A}\left(E=E^{H}\right)=\underline{U}+2\left(W^{L H}-\bar{W}\right) p_{H}\left(1-p_{H}\right)>\underline{U} \text {, si } W^{L H}>\bar{W}
$$

Nótese que para un nivel de incentivos $W^{L H}=\bar{W}$, el agente obtendría la misma utilidad cooperando que actuando de manera individual (se situaría en el nivel de utilidad de reserva). Es decir, bajo este esquema de incentivos los agentes innovadores estarían en una situación de indiferencia entre ambos escenarios, ceteris paribus.

Por lo tanto, para que el gobierno pueda inducir la cooperación entre agentes, este nivel de incentivos, $W^{\mathrm{LH}}$, debe ser tal que $W^{\mathrm{LH}}>\bar{W}$, esto es, debe situarse por encima de los incentivos medios asociados con incentivos altos $W^{H}$ y bajos $W^{L}$ respectivamente. ¿Cuál es la racionalidad económica de esta estructura de incentivos? La cooperación entre agentes, con independencia de su naturaleza, causa un potencial problema de riesgo moral (Holmström, 1982; Macho-Stadler y Pérez-Castrillo, 1991; 1993). En otras palabras, algunos agentes pueden asumir un nivel de esfuerzo bajo cuando cooperan con otros, lo que perjudica a los agentes que asumen esfuerzos elevados durante la cooperación cuando el gobierno no es capaz de discriminar la remuneración de acuerdo al esfuerzo realizado —nótese que las acciones de los agentes, su esfuerzo, no es observable 
directamente- Dada esta situación, para estimular la cooperación entre agentes es necesario ofrecer incentivos que compensen o atenúen el costo derivado del potencial comportamiento de riesgo moral que surge de la cooperación. Dicho estímulo se logra si los incentivos $W^{\mathrm{LH}}$, que se establecen en el caso de que el resultado de las actividades de I+D es $R^{L H}$, cumplen la condición de que $W^{L H}$ $>\bar{W}$. Con esta estructura de incentivos se verifica que la utilidad espera del agente en un escenario de cooperación es mayor que cuando los agentes actúan individualmente; así, los agentes obtiene beneficios de la cooperación en actividades de I+D. Además, estas ganancias serán tanto mayores cuanto mayor sea la diferencia entre $W^{L H}$ y $\bar{W}$. Se puede concluir que la política tecnológica del gobierno puede estimular la cooperación en actividades de $\mathrm{I}+\mathrm{D}$ mediante un adecuado diseño del programa de incentivos.

\section{CONCLUSIONES, LimitACIONES Y FUTURAS LÍNEAS DE INVESTIGACIÓN}

En este trabajo se ha analizado, desde un enfoque teórico-formal, la capacidad de la política tecnológica para promover el comportamiento cooperativo entre los agentes que desarrollan actividades de I+D. Para ello, a partir de los fundamentos del modelo principal-agente hemos estudiado la relación que se establece entre un principal - en este caso, el gobierno, responsable de la elaboración del programa de política tecnológica-y los agentes —empresas, universidades y organizaciones que realizan actividades de $\mathrm{I}+\mathrm{D}$ - con el objetivo de determinar la estructura de incentivos que el gobierno debe diseñar con la finalidad de promover la cooperación. Los resultados analíticos ponen de manifiesto la existencia de un cierto umbral de incentivos bajo el que la cooperación en actividades de $\mathrm{I}+\mathrm{D}$ entre agentes puede promoverse. Por lo tanto, se prueba que la política tecnológica puede producir variaciones en el comportamiento de los agentes en lo referente al desarrollo de actividades de I+D.

\section{Implicaciones teóricas}

Una de las implicaciones de este trabajo es que ofrece una nueva perspectiva de investigación teórico-analítica en el ámbito de la política tecnológica y la cooperación en actividades de I+D. Hasta donde nosotros sabemos, los trabajos realizados en este terreno concreto se han situado en el plano puramente em- 
pírico. De este modo, el estudio de este fenómeno mediante el uso de modelos formales, contribuye a enriquecer y sistematizar dicho ámbito de estudio. En esta línea, y en formato todavía borrador, existe un trabajo que incorpora una visión formal de la relación entre la política tecnológica y la innovación utilizando la teoría de juegos evolutiva y que, por tanto, contribuye a ir desarrollando e incorporando un planteamiento más sistematizado al estudio de dicha relación (Quynh Le, Nakahira y Mikami, 2009). Es nuestro cometido, profundizar la investigación mediante un enfoque más formal que nos permita alcanzar una mayor comprensión de la relación entre la política tecnológica y las actividades de $\mathrm{I}+\mathrm{D}$ desarrolladas por los agentes.

\section{Implicaciones para la política}

Algunas implicaciones que pueden derivarse de nuestros resultados, desde un punto de vista práctico, son las siguientes: en primer lugar, los gobiernos, mediante el diseño de un sistema adecuado de incentivos para actividades de $\mathrm{I}+\mathrm{D}$ pueden estimular la cooperación. En particular, incentivos elevados (e.g., altos subsidios) atenúan los costos de la colaboración que están relacionados con el problema de riesgo moral y los costos de coordinación entre agentes, facilitando así la cooperación. En segundo lugar, y relacionado con la implicación anterior, la cuantía o volumen de estos subsidios públicos a las actividades de $\mathrm{I}+\mathrm{D}$, especialmente en aquellos programas en los que la cooperación es un requisito sine qua non, puede propiciar comportamientos oportunistas - captura de rentas-. En otras palabras, si los programas ofrecen una financiación muy generosa, esta puede constituir un objetivo en sí misma y, de este modo, los agentes cooperan principalmente para capturar rentas y no porque tengan una voluntad verdadera de cooperar. Además, este comportamiento puede influir en el logro de los objetivos tecnológicos previstos en tales proyectos, generando una cooperación artificial que no garantiza su correcto desarrollo, dada la falta de un genuino y auténtico interés en su conclusión.

Relacionado con lo anterior, varios trabajos (e.g., Heijs, 2003) ponen de manifiesto la existencia de un problema de free-riding relacionado con la concesión de subsidios públicos con bajo interés para el desarrollo de actividades de I+D, mostrando la evidencia de que los beneficiarios de tales préstamos pueden llevar a cabo el mismo nivel de actividades de $\mathrm{I}+\mathrm{D}$ sin las ayudas públicas y que estos agentes pueden alcanzar también un nivel inferior en cuanto a los objetivos tecnológicos y comerciales propuestos. 
En tercer lugar, y desde una perspectiva dinámica, los programas públicos de ayudas a actividades de $\mathrm{I}+\mathrm{D}$ para promover acuerdos de colaboración pueden ajustarse de acuerdo con la relación existente entre los colaboradores y, en este sentido, contribuir a una mejor optimización social en la asignación de recursos para las actividades tecnológicas. En otras palabras, en el caso de que los agentes mantengan una cooperación sostenida a lo largo del tiempo, no sería necesario mantener un nivel de incentivos tan elevado como en el caso en el que la cooperación fuese incipiente, consecuencia de que la reputación y conocimiento adquiridos a lo largo de la relación podría atenuar los costos iniciales de la cooperación mencionados anteriormente (riesgo moral). Esto también contribuye a mitigar el sesgo de auto-selección en la participación de programas públicos de ayudas a la $\mathrm{I}+\mathrm{D}$, puesto que, en el caso de que estos agentes ya hubieran recibido fondos y estuvieran implicados en procesos de cooperación durante un largo periodo de tiempo, tendrían ventajas adicionales para participar otra vez en estos programas, en comparación con aquellos otros agentes que nunca hubieran colaborado. Finalmente, se puede advertir que la eficiencia del programa de incentivos para estimular la cooperación tecnológica depende de la existencia de una cultura cooperativa. En este sentido, en entornos con una mayor predisposición al trabajo colaborativo, la cantidad de incentivos necesarios para estimular la cooperación podría ser inferior a la que corresponda a aquellos entornos donde la cultura cooperativa esté menos implantada.

\section{Limitaciones y futuras líneas de investigación}

Evidentemente todo trabajo está sometido a limitaciones, las cuales a su vez constituyen nuevas oportunidades para avanzar y profundizar en la investigación. Por lo que respecta a las limitaciones de nuestro modelo, hemos considerado que los agentes sólo pueden inferir perfectamente el esfuerzo y comportamiento que cada uno ha aplicado una vez concluido el proyecto y evaluado el resultado. Esta limitación abre la posibilidad a un nuevo desarrollo teórico y extensión del modelo, introduciendo un mecanismo de supervisión ex ante, bien de carácter interesado o desinteresado (supervisión interna costosa). Esto es, en dicha supervisión se pueden considerar varios mecanismos, desde la supervisión interna o control mutuo hasta la supervisión externa realizada por algún agente especializado. Por otro lado, los resultados que se obtienen de nuestro modelo podrían contrastarse empíricamente. De este modo, podría 
estudiarse la existencia de un cierto umbral de ayudas públicas a la I+D a partir del cual la cooperación se incrementa. A partir de esta verificación previa, otra posible extensión empírica podría ser un estudio comparativo entre agentes de diferentes países para evaluar si el umbral de ayudas públicas a las actividades de $\mathrm{I}+\mathrm{D}$ varía entre ellos; en caso de existir diferencias, estas podrían ser un indicador de la influencia de factores culturales que condicionan la tendencia a la cooperación.

\section{REFERENCIAS}

Bayona, C., García-Marco, T. y Huerta, E., 2003. ¿Cooperar en I+D? Con quién y para qué. Revista de Economía Aplicada, XI(31), pp. 103-134.

Belderbos, R., Carreeb, M. y Lokshinb, B., 2004. Cooperative R\&D and Firm Performance. Research Policy, 33, pp. 1477-1492.

Buisseret, T.J., Cameron, H. y Georghiou L., 1995. What Difference does it Make? Additionality in the public support of R\&D in large firms. International Journal of Technology Management, 10, pp. 587-600.

Buson, I. y Fernández-Ribas, A., 2008. The Impact of Participation in R\&D Programs on R\&D Partnerships. Research Policy, 37(2), pp. 240-257.

Davenport, S., Grimes, C. y Davies, J., 1998. Research Collaboration and Behavioral Additionality: A New Zealand case study. Technology Analysis and Strategic Management, 10(1), pp. 55-67.

Fölster, S., 1995. Do Subsidies to Cooperative R\&D Actually Stimulate R\&D Investment and Cooperation. Research Policy, 24(3), pp. 403-417.

Hagedoorn, J., 2002. Inter-firm R\&D partnerships: An overview of major trends and patterns since 1960. Research Policy, 31, pp. 477-492.

Hagedoorn, J., Link, A.N. y Vonortas, N.S., 2000. Research Partnerships. Research Policy, 29, pp. 567-586.

Heijs, J., 2003. Frerider Behaviour and he Public Financing of R\&D Activities in Enterprises: The case of the Spanish low interest credits for R\&D. Research Policy, 32(3), pp. 445-461.

Holmström, B., 1982. Moral Hazard in Teams. Bell Journal of Economics, 13(2), pp. 324-340.

Macho-Stadler, I. y Pérez-Catrillo, J.D., 1991. Moral Hazard and Cooperation. Economic Letters, 53(1), pp. 17-20. 
Macho-Stadler, I. y Pérez-Catrillo, J.D., 1993. Moral Hazard with Several Agents: The gains from cooperation. International Journal of Industrial Organization, 11, pp. 73-100.

Miotti, L. y Sachwald, F., 2003. Cooperative R\&D: Why and with whom? An integrated framework analysis. Research Policy, 32(8), pp. 1481-1499.

Mohnen P. y Hoareau, C., 2003. What Type of Enterprises Forges Closes Links with Universities and Government Labs? Evidence from the CIS2. Managerial and Decision Economics, 24, pp. 133-145.

Nicholson, W., 2005. Microeconomic Theory: Basic principles and extensions. 9a edición. Estados Unidos: Thomson/South-Western.

OECD (Organisation for Economic Co-operation and Development), 2002. Science, Technology and Industry Outlook 2002. Disponible en: <http://www. oecd.org/sti/inno/oecdsciencetechnologyandindustryoutlook2002.htm>. OECD, 2010. Measuring Innovation. A New Perspective. Paris: OECD [Versión en línea disponible en: <www.oecd.org/innovation/strategy/measuring >].

Quynh Le, L.T., Nakahira, K.T. y Mikami, Y., 2012. Analysis on the Impact of Government Policies towards Technological Innovation Using Evolutionary Game Theory, retrieved from < http:/ /www.econ.hit.ac.jp/ aokada/kakengame/meeting/workshop/paper2012/Lien.pdf $>$. 
